\title{
Are Transrectal Prostate Biopsies Routinely Indicated in Patients with Incidentally Diagnosed Prostate Cancer following Transurethral Resection of the Prostate for Benign Disease?
}

\author{
Lui Shiong Lee $^{\mathrm{a}}$ Suntharasivam Thiruneelakandasivam ${ }^{\mathrm{a}}$ Matthew K. Hong ${ }^{\mathrm{f}, \mathrm{g}}$ \\ Justin S. Peters ${ }^{f, g}$ Anne Warren ${ }^{b}$ Rob Mills ${ }^{c}$ David Greenberg ${ }^{d}$ \\ Karen Wright ${ }^{d}$ Vincent Gnanapragasam ${ }^{\mathrm{e}}$ \\ Departments of a Urology and b ${ }^{b}$ Pathology, Addenbrookes Hospital, Cambridge, ' Department of Urology, Norfolk \\ and Norwich University Hospital, Norwich, d Eastern Cancer Registration and Information Centre, Cambridge, \\ and ${ }^{\mathrm{e}}$ Department of Oncology, Hutchison/MRC Centre, University of Cambridge, Cambridge, UK; ${ }^{\mathrm{f}}$ Department \\ of Surgery, University of Melbourne, Royal Melbourne Hospital, and ${ }^{9}$ Australian Prostate Cancer Research Centre \\ Epworth, Melbourne, Vic., Australia
}

\section{Key Words}

Prostatectomy - Needle biopsy - Transurethral prostate resection · Surgical pathology · Gleason grading

\begin{abstract}
Objective: To determine the indication of routine transrectal ultrasound-guided needle biopsy (TRUSBX) of the prostate gland following incidental cancer diagnosis after transurethral resection of the prostate (TURP) for benign prostatic hyperplasia. Materials and Methods: A multi-institutional search identified 63 patients with incidental TURP-diagnosed prostate cancer from 2001 to 2010, who underwent subsequent TRUSBx or radical prostatectomy (RP). The Gleason scores from TURP were compared to those from TRUSBX or RP. Whole mount maps from RP were analysed to provide an anatomical basis for the correlation observed. To determine the clinical impact of this problem, the incidence of TURP-diagnosed prostate cancer in the population was also determined. Results: Of 22 patients who underwent TRUSBX, the rates of Gleason score concordance, upgrading and downgrading were 32, 14 and 54\% respectively (Spearman
\end{abstract}

correlation coefficient 0.20 ). Most cases of pathological downgrading consisted of benign cores at biopsy. Therefore, TRUSBx did not give additional Gleason score (GS) information in $86 \%$ of patients. Of $41 \mathrm{RP}$ patients, the respective rates were 61, 22 and 17\% (Spearman correlation coefficient 0.15 ). The majority of them retained a similar or lower GS between TURP and RP. Of 13 whole mount maps analysed, $6(46 \%)$ were found with anterior/transitional zone (AZ/TZ) tumours, 6 (46\%) with multifocal tumours and 1 (8\%) with a large peripheral zone (PZ) tumour extending into the TZ. Regional population data show that despite a gradual reduction in the proportion of TURP-diagnosed cases over the past decade, they still account for $8.5-13 \%$ of all new cases. Conclusion: TURP-diagnosed prostate cancers represent predominantly AZ tumours. A TRUSBx does not give additional GS information in a majority of cases, and therefore is not routinely indicated. It may be selectively useful prior to active surveillance, but not in all pursuing radical treatment. These findings may help reduce unnecessary TRUSBx in the population.

(c) 2013 S. Karger AG, Basel

\section{KARGER}

E-Mail karger@karger.com

www.karger.com/uin
(C) 2013 S. Karger AG, Basel

0042-1138/13/0914-0397\$38.00/0
Vincent Gnanapragasam

Uro-oncology Group, Department of Oncology

Hutchison/MRC Centre, University of Cambridge Hills Road, Cambridge CB2 0XZ (UK)

E-Mail vincent.gnanapragasam@addenbrookes.nhs.uk 


\section{Introduction}

Transurethral resection of the prostate (TURP) remains the gold standard of surgical treatment for symptomatic bladder outlet obstruction secondary to benign prostatic hyperplasia (BPH) [1]. The contemporary prevalence of prostate cancer incidentally found at TURP is variable, accounting for $7-17 \%$ of all diagnosed cases of prostate cancer in selected populations [2-6]. This observation is concordant with pathological mapping studies demonstrating the peripheral zone (PZ) as the primary focus of prostatic adenocarcinoma in a majority of cases [7-9]. Therefore, following an incidental diagnosis of prostate cancer after TURP, it is not uncommon for urologists to advocate additional transrectal ultrasound-guided needle biopsy (TRUSBx) in an attempt to provide a more representative assessment of the extent and grade of cancer present. There are however no guidelines relating to best clinical practice in this situation, leading to a widespread variation in clinical management. Although TRUSBx may be performed easily and safely in the clinic, there are procedure-related morbidities such as bleeding and urinary infections which may be potentially lifethreatening $[10,11]$. In addition, it is not clear whether such additional procedures add value to clinical decisionmaking. To date there are no publications on this topic, making an accurate perspective to this clinical problem difficult to achieve. Existing publications focus on patients who were screened with a TRUSBx prior to TURP, but they do not discuss the role of TRUSBx after a TURPbased cancer diagnosis [12-14]. In this study, we compared the histological findings from primary adenocarcinoma of the prostate incidentally diagnosed at TURP performed for $\mathrm{BPH}$ with corresponding samples obtained from a subsequent TRUSBx or radical prostatectomy (RP). In cases where whole mount maps of the prostate gland were available after RP, the location and size of the tumour lesions were assessed to elucidate a possible anatomical basis that might account for the pathological correlations identified.

\section{Materials and Methods}

A multicentre search was initiated to identify patients from prospectively maintained cancer registries in the UK and Australia. The period of study was 2001-2010. Patients who had a subsequent TRUSBx following incidental prostate cancer diagnosis were identified from interrogation of the prostate cancer records of the UK Eastern Cancer Registration and Information Centre (ECRIC). Patients who had a RP following diagnosis by TURP were identified from the Departments of Urology, Addenbrookes
Hospital (Cambridge), Norfolk and Norwich Hospital and Royal Melbourne Hospital (Australia). Only TRUSBx or RP procedures performed within 18 months of TURP were accepted so as to maximise the detection of any missed synchronous tumours. We also focused on men aged 75 or less so as to specifically assess men who would most likely be eligible for radical therapy. Men with a known history of prostate cancer were excluded, as were men who had TRUSBx prior to TURP. The variables analysed included patient demographics, preoperative prostate-specific antigen (PSA), pathological primary and secondary GS, weight of resected tissue (from TURP), number of cores taken during TRUSBx, and location of tumour lesions (based on whole mount slides after RP). Tumour stage was not assessed in this study. Statistical analysis was performed using Spearman correlation coefficient and Wilcoxon rank-sum test with significance defined at $\mathrm{p}<0.05$. In this study, pathological GS groups were classified into those with a GS of 6, 7 and $\geq 8$. Pathological up- or downgrading of the GS was defined as a change from one GS score group to another [15]. Finally, to gain an accurate perspective on the clinical impact of this topic, we further interrogated the ECRIC database to define the prevalence of TURP-diagnosed prostate cancer in the Eastern England Cancer Network over the last decade.

\section{Results}

\section{Patient Demographics}

There were 63 suitable patients identified for this study from our multi-institutional cohort search. The mean age of the patients was 63 years (range 47-73) and the mean preoperative PSA level was $6.8 \mathrm{ng} / \mu \mathrm{l}$ (range $0.3-41$ ). Most patients were found to have tumours with pathological GS $6(59 \%, 37 / 63)$, followed by GS $7(38 \%, 24 / 63)$ and GS $8(3 \%, 2 / 63)$ (table 1$)$. The mean prostate weight resected at TURP was $23.6 \mathrm{~g}$ (range 2.4-93). The pathological stage was T1a in 54\% (34/63) of patients and T1b in the remaining cases ( 1 patient with missing variable). Following TURP, 35\% (22/63) and 65\% (41/63) of patients underwent subsequent TRUSBx and RP respectively. There were $11 \%(7 / 63)$ of patients who underwent TRUSBx followed by RP.

\section{Comparison of Pathology between TURP and TRUSBX}

We first tested the yield of a subsequent TRUSBx following TURP cancer diagnosis. For patients who underwent TRUSBx, a mean of 12 (range 7-19) cores were obtained at the procedure, which was performed at a mean interval of 8.3 months (range 1-18) following TURP. The concordance rate of GS between TURP and TRUSBx was 32\% (7/22) (Spearman correlation coefficient 0.20 ) (table 2). On the other hand, $54 \%$ of cases (12/22) were pathologically downgraded on TRUSBx, and $14 \%(3 / 22)$ cases were upgraded. In 10 cases of path- 
Table 1. Patient characteristics

$\mathrm{p}$ value

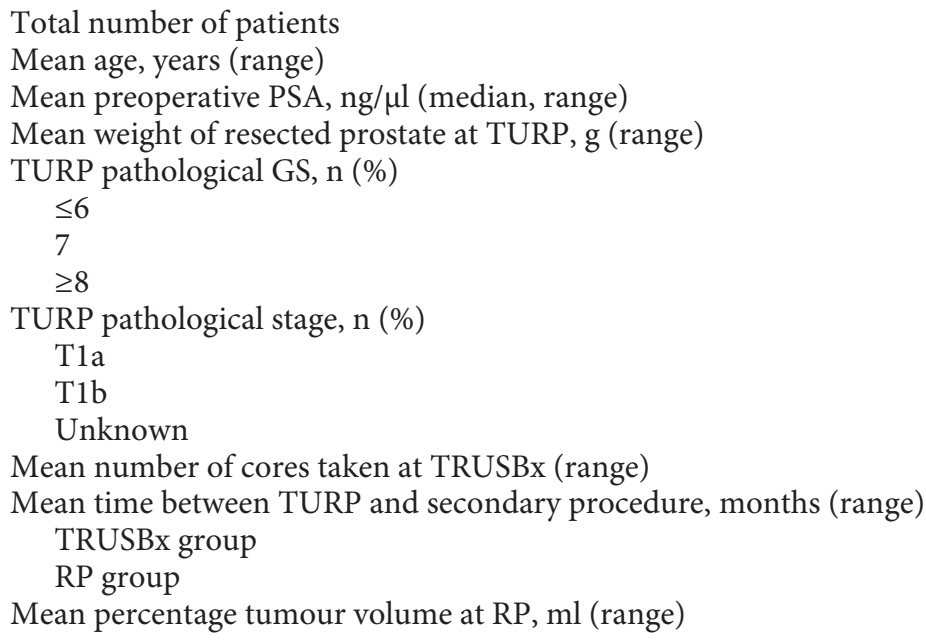

$\begin{array}{ll}63 & \\ 63(47-73) & \text { NA } \\ 6.8(4.1,0.3-41) & \text { NA } \\ 23.6(2.4-93) & \text { NA } \\ 37(59) & \text { NA } \\ 24(38) & \\ 2(3) & \\ 34(54) & \text { NA } \\ 28(44) & \\ 1(2) & \\ 12(7-19) & \text { NA } \\ 8.3(1-18) & 0.64 \\ 7.2(2-18) & \\ 10.8(1-50) & \text { NA }\end{array}$

ological downgrading there was no cancer detected at TRUSBx. Therefore, in $86 \%$ (19/22) of these patients, a subsequent TRUSBx did not yield additional new pathological grade information. There were 7 patients who underwent both TRUSBx and RP. Three patients had a concordant GS between TURP and RP. In these three cases, an additional TRUSBx would not have provided additional information regarding the final GS. In 4 patients with discordant GS between TURP and TRUSBx, only 1 case demonstrated pathological upgrading at TRUSBx, which was confirmed at RP. Therefore, in this small subset of patients, an intervening TRUSBx between TURP and RP provided useful GS information in only $15 \%$ of individuals (1/7) (online suppl. table 1, www.karger.com/doi/10.1159/000350898).

\section{Comparison of Pathology between TURP and RP}

We next repeated the analysis in patients who underwent RP after TURP. Here we were specifically interested in tumour grading as well as tumour location. $61 \%(25 / 41)$ of men had concordant GS between TURP and RP (Spearman correlation coefficient 0.15 ) (table 3 ). The rates of pathological up- and downgrading were $22 \%(9 / 41)$ and $17 \%$ (7/41) respectively. The majority of cases therefore retained a similar or lower GS between TURP and RP.

Whole mount maps from RP specimens were available in 13 matched cases, which allowed analysis of tumour location and volume of TURP-detected cancers. In these patients, 70\% (9/13) had a concordant GS comparing
Table 2. Pathological GS correlation between TURP and TRUSBx samples $(\mathrm{n}=22)$

\begin{tabular}{llll}
\hline TURP GS & $\mathrm{n}(\%)$ & Corresponding TRUSBx GS, n \\
\hline 6 & \multirow{2}{*}{$17(77)$} & no cancer detected & 5 \\
& & HGPIN & 2 \\
& & GS 6 & 7 \\
& & GS 7 & 2 \\
7 & & GS 8 & 1 \\
& & no cancer detected & 3 \\
8 & $4(18)$ & GS 6 & 1 \\
& & GS 7 & 1 \\
\hline
\end{tabular}

HGPIN = High-grade prostatic intraepithelial neoplasia.

Table 3. Pathological GS correlation between TURP and RP samples $(\mathrm{n}=41)$

\begin{tabular}{lllc}
\hline TURP GS & $\mathrm{n}(\%)$ & \multicolumn{2}{l}{ Corresponding RP GS, n } \\
\hline 6 & $20(49)$ & GS 6 & 11 \\
& & GS 7 & 9 \\
7 & $20(49)$ & GS 6 & 6 \\
8 & $1(2)$ & GS 7 & 14 \\
& & GS 7 & 1 \\
\hline
\end{tabular}




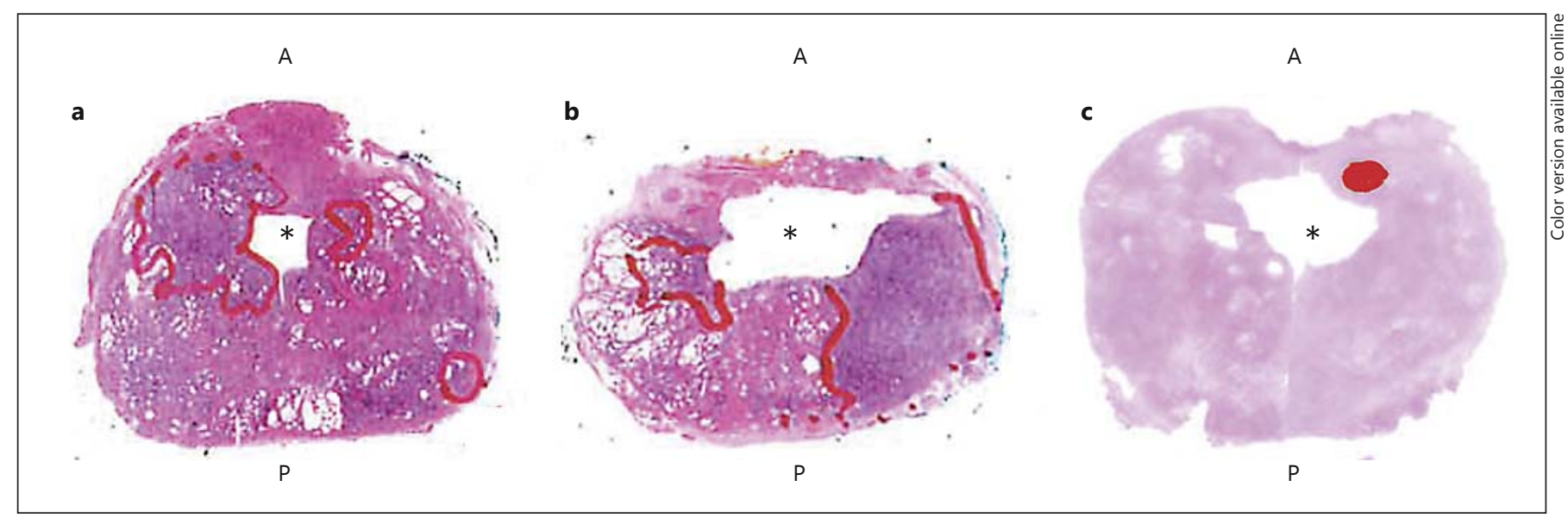

Fig. 1. Whole mount maps from RP samples. a Multifocal tumours in the AZ, TZ and PZ. b Large PZ tumour extending into the TZ. c Primary TZ tumour. TURP cavities are seen in all specimens (indicated by an asterisk) and indicate the location of the TZ. Tumours are highlighted in red. $\mathrm{A}=$ Anterior; $\mathrm{P}=$ posterior.
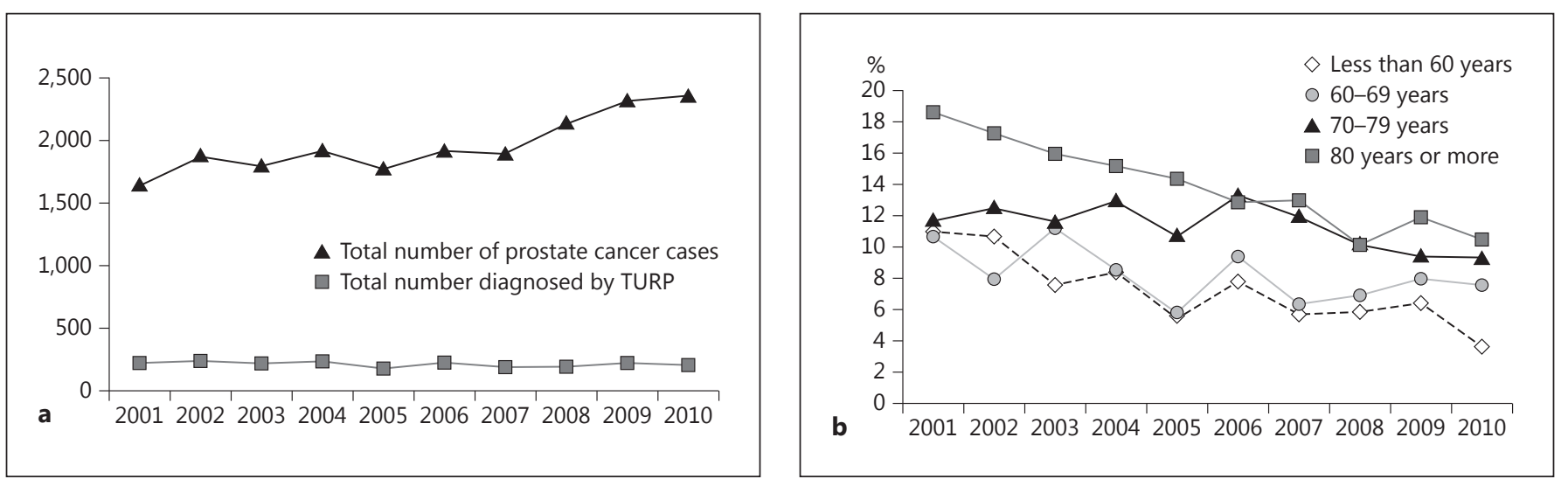

Fig. 2. a Incidence of prostate cancer and prostate cancer diagnosis by TURP in East Anglia from 2001 to 2010. b Proportion of TURP-diagnosed cancers in East Anglia from 2001 to 2010, stratified by age.

TURP and RP. Of these 9 patients, 5 had tumours arising from the anterior zone (AZ) and transitional zone (TZ), 3 had multifocal tumours in both $\mathrm{PZ}$ and $\mathrm{AZ}$, and 1 had a large PZ tumour extending into the TZ. Of the 4 patients with discordant GS the tumour locations were multifocal in 3 cases and TZ in 1 case. Therefore in 6 out of 13 cases, the primary tumour was found to be anteriorly located and largely in the TZ (fig. 1).

\section{Contemporary Incidence of TURP-Diagnosed Prostate Cancer}

To define the clinical relevance of our study, we interrogated the ECRIC data registry to investigate the contemporary trends in primary cancer detection by TURP.
In this analysis we observed an increasing incidence of prostate cancer from 2001 to 2010 . In contrast the number of cases of prostate cancer diagnosed via TURP has stayed relatively constant (approximately 200 cases per annum), but has decreased as a proportion of total cases $(13.2 \%$ in 2001 vs. $8.5 \%$ in 2010 ) (fig. 2a). When stratified by age groups, however, the proportions of TURP-diagnosed cancers at each age tier remained fairly constant throughout the decade. The only exception was in the $<60$ years cohort where there was a sustained decrease in more recent years (fig. 2b). These data suggest that TURP-diagnosed tumours constitute about $10 \%$ of all cancers diagnosed. As such the issue of optimal investigation of these men remains a relevant topic, particularly in older men. 


\section{Discussion}

To our knowledge, this is the first study looking at the pathological correlation between Gleason grading at TURP and subsequent TRUSBx or RP. This cohort was confined to patients with no clinical suspicion of prostate cancer at the time of TURP. Clearly, individuals in this study are distinct from those where TURP was utilised as a modality of TZ biopsy following negative PZ biopsies, as the latter looked at patients with high indices of suspicion for prostate cancer $[2,16]$. This study is also not intended to look at TURP as an alternative means to TRUSBx for primary diagnosis of prostate cancer generally.

Although TURP is not the predominant technique for detection of prostate cancer, population-based data from the region show that the prevalence of TURP-diagnosed cancer is not insignificant and would constitute up to $10 \%$ of all newly diagnosed cases. The ability for better discrimination in patient selection for secondary TRUSBx would still have considerable impact on healthcare resources and procedural morbidities (especially in the elderly population). Hence, establishing guidance for this approach would be a useful exercise. Interestingly, the relative rates of TURP-diagnosed cancers are fairly similar in other series from distinct patient populations [17]. Therefore, the yield from this study may potentially be extrapolated to other populations.

Given that the rate of pathological upgrading from TURP to TRUSBx is low (14\% in this study), a routine secondary TRUSBx would possibly be useful in patients with apparent low-grade disease contemplating active surveillance (AS) or even watchful waiting. This may help identify the small proportion of men who actually harbour higher-grade tumours that would preclude AS. A re-biopsy in men who are diagnosed with GS 7 or above disease however is probably unnecessary. If RP is being considered, then a routine TRUSBx intended to detect higher-grade tumours is again unnecessary as surgery effectively removes the entire prostate gland for pathologi$\mathrm{cal}$ analysis. Other authors have also reported low rates of pathological upgrading (from TRUSBx to RP) that range from 7 to $30 \%[18,19]$. This would lend further support to a selective role for TRUSBx only prior to consideration of a conservative management option. Where patients are being treated with radical radiation, a TRUSBx also may be selectively indicated in those with low GS, where subsequent detection of a high-grade tumour might influence the duration and administration of neoadjuvant androgen deprivation [20-22].

TRUSBx in Incidentally Diagnosed Prostate Cancer following TURP for BPH
A peripherally directed TRUSBx may help determine the feasibility of a nerve-sparing procedure. This study demonstrated that TURP-diagnosed cancers tend to be localised to the AZ and TZ (46\% of cases with whole mount maps). In contrast, previous mapping studies have demonstrated the $\mathrm{PZ}$ as the predominant site of tumourigenesis in primary prostate adenocarcinoma [7, 9]. TURP-diagnosed cancers may therefore represent a group of cancers with a distinct anatomical mapping location, as compared to those conventionally detected through prostate needle biopsies. Given this information, it is reasonable to postulate that following a TURP diagnosis of prostate cancer, a routine peripherally directed TRUSBx would yield negative cores in half the time. This is, in fact, consistent with the observation in our TRUSBx cohort where there was a $46 \%$ incidence of negative biopsies. Furthermore, looking at the cohort of patients who underwent all three procedures (TURP, TRUSBx, RP), routine TRUSBx provided very limited additional information towards prediction of the final GS (at RP).

Nerve sparing is normally advocated for low GS tumours and it would be reasonable to suggest that a routine TRUSBx is not required for these men [23]. However, the presence of significant tumour volume in the prostate, as implied by a large tumour burden in the resected TURP tissue, may prompt a TRUSBx prior to RP. This may help detect significant $\mathrm{PZ}$ disease that may compromise surgical margins during nerve-sparing RP. In this instance however staging information by magnetic resonance imaging may equally be informative on tumour laterality and extent. Where GS $\geq 7$ disease is diagnosed, a TRUSBx may be useful to identify the anatomical extent of the tumour and the laterality of the lesion. In this context however imaging may again be able to provide this information without the need for a repeat biopsy. Certainly ongoing improvements in imaging techniques will allow for precise determination of tumour location, size and the presence of extracapsular extension [24-26]. However, we also recognise that existing imaging modalities (such as magnetic resonance imaging) may have limited resolution in a postsurgical field, especially in the assessment of tumour burden in the remnant prostate. Yet, given that TURP resects tissue in the TZ only, the determination of capsular involvement should not be affected. In the future, higher-resolution imaging may overcome existing limitations and this may obviate the need for a biopsy altogether.

In this study, there were no patients with pT0 disease (no residual disease) at RP. In contrast, several other publications in the literature report pT0 rates of $2-48 \%$ 
[12-14]. Some patients in these studies had a TRUSBx prior to TURP, and those with a positive histology were subsequently treated via RP. Thus, these preceding studies have excluded patients with significant $\mathrm{PZ}$ disease (detectable by TRUSBx), while selecting for patients with predominant AZ/TZ tumours. To our knowledge, these studies do not describe TRUSBx after a TURP diagnosis of cancer and prior to RP. Therefore, the relative strength of our study is the inclusion of an 'unscreened' population that more closely approximates actual clinical practice most patients do not receive a TRUSBx prior to TURP for $\mathrm{BPH}$.

The limitations of this study include the difficulty in identification of a sufficient number of suitable patients. Despite a multicentre search strategy, the study cohort remained small, which is reflective of the limited prevalence of these patients in the population. Therefore, the only plausible manner by which sufficient patient numbers can be accrued would be via a prospective multicentre collaborative study. The existing database might not have captured patients who did undergo secondary procedures in a different institution within 18 months of TURP diagnosis. Other limitations of this study include its retrospective non-randomised nature, and therefore we accept the presence of a selection bias contributing to the choice of the secondary procedure (TRUSBx versus upfront RP). The RP group may also be inclined towards patients with localised disease, although all patients in this study had clinical stage T1c at diagnosis. Nevertheless, comparison of the baseline characteristics of the study cohort shows no obvious differences as compared to those reported in other published surgical series [27, 28]. There is also a lack of central pathological review. The retrospective nature of this study also does not allow for standardisation of the timing and extent of TRUSBx. We recognise that the quality of tissue sampling is related to the number of cores taken, and this is related to a patient's prostate volume and the clinician's preference. There is also no reliable means to establish the actual site of the prostate gland sampled during TRUSBx, but it would be reasonable to assume peripherally directed biopsies, especially in the presence of a TURP cavity.

We defined a time limit of 18 months between initial TURP diagnosis and subsequent TRUSBx. That would give a balance between the need for a re-biopsy versus the risk of tumour progression (while on surveillance). This time period also defined the window that allowed the capture of re-biopsy events in this retrospective review. Specifically, there were no guiding publications in the existing literature that would, otherwise, suggest a more ap- propriate time interval. Therefore, we drew inference from existing AS protocols, for which there is no consensus between institutions [29-32]. For instance, patients on AS may undergo scheduled re-biopsies as early as 12 months post diagnosis, or only when triggered by an event (such as a rise in serum PSA levels). Thus, the stipulated time interval also allowed for existing variation in AS protocols. Finally, PSA data in the interval between TURP and re-biopsy or RP were also not available for the majority of men and hence we have not considered this variable in the study.

In conclusion, this study is the first to investigate the usefulness of a subsequent TRUSBx following the diagnosis of incidental prostate cancer after TURP. The findings of this study do not suggest that routine TRUSBx is indicated after a TURP diagnosis of cancer. Within the limits of our sample size and retrospective cohort, we suggest that a TRUSBx is indicated in low GS cancers where a decision for conservative management is being contemplated. For higher GS, the value of a TRUSBx is doubtful, particularly in the context of whole gland therapy. In men electing surgical management with nerve-sparing RP, the role of a TRUSBx remains unclear. In this context however improvements in tumour location and characterisation via non-invasive standard and functional magnetic resonance imaging may be an equally valid strategy which would avoid the need for a biopsy [24]. Further studies are needed to test and corroborate these findings and are likely to need multicentre collaboration.

References

\footnotetext{
Reich O, Gratzke C, Stief CG: Techniques and long-term results of surgical procedures for BPH. Eur Urol 2006;49:970-978; discussion 978.

2 Zigeuner R, Schips L, Lipsky K, et al: Detection of prostate cancer by TURP or open surgery in patients with previously negative transrectal prostate biopsies. Urology 2003;62: 883-887.

-3 Andrèn O, Garmo H, Mucci L, et al: Incidence and mortality of incidental prostate cancer: a Swedish register-based study. Br J Cancer 2008;100:170-173.

-4 Merrill RM, Wiggins CL: Incidental detection of population-based prostate cancer incidence rates through transurethral resection of the prostate. Urol Oncol 2002;7:213-219.

5 Ornstein DK, Rao GS, Smith DS, Andriole GL: The impact of systematic prostate biopsy on prostate cancer incidence in men with symptomatic benign prostatic hyperplasia undergoing transurethral resection of the prostate. J Urol 1997;157:880-883; discussion 883-884.
} 
$\checkmark 6$ Dellavedova T, Ponzano R, Racca L, et al: Prostate cancer as incidental finding in transurethral resection. Arch Esp Urol 2010;63: 855-861.

77 McNeal JE: Origin and development of carcinoma in the prostate. Cancer 1969;23:24-34.

8 McNeal JE: The zonal anatomy of the prostate. Prostate 1981;2:35-49.

$>9$ Chen ME, Johnston DA, Tang K, et al: Detailed mapping of prostate carcinoma foci: biopsy strategy implications. Cancer 2000;89: 1800-1809.

10 Djavan B, Waldert M, Zlotta A, et al: Safety and morbidity of first and repeat transrectal ultrasound guided prostate needle biopsies: results of a prospective European prostate cancer detection study. J Urol 2001;166:856860.

11 Ghani KR, Dundas D, Patel U: Bleeding after transrectal ultrasonography-guided prostate biopsy: a study of 7-day morbidity after a six-, eight- and 12-core biopsy protocol. BJU Int 2004;94:1014-1020.

$>12$ Magheli A, Rais-Bahrami S, Carter HB, et al: Subclassification of clinical stage T1 prostate cancer: impact on biochemical recurrence following radical prostatectomy. J Urol 2007; 178:1277-1280; discussion 1280-1281.

$>13$ Masue N, Deguchi T, Nakano M, et al: Retrospective study of 101 cases with incidental prostate cancer stages T1a and T1b. Int J Urol 2005;12:1045-1049.

-14 Melchior S, Hadaschik B, Thüroff S, et al: Outcome of radical prostatectomy for incidental carcinoma of the prostate. BJU Int 2009;103:1478-1481.
15 Epstein JI, Feng Z, Trock BJ, Pierorazio PM: Upgrading and downgrading of prostate cancer from biopsy to radical prostatectomy: incidence and predictive factors using the modified Gleason grading system and factoring in tertiary grades. Eur Urol 2012;61:1019-1024.

16 Kim DK, Kim SJ, Moon HS, et al: The role of TURP in the detection of prostate cancer in $\mathrm{BPH}$ patients with previously negative prostate biopsy. Korean J Urol 2010;51:313-317.

17 Tombal B, De Visccher L, Cosyns JP, et al: Assessing the risk of unsuspected prostate cancer in patients with benign prostatic hypertrophy: a 13-year retrospective study of the incidence and natural history of T1a-T1b prostate cancers. BJU Int 1999;84:1015-1020.

18 Lowe BA, Barry JM: The predictive accuracy of staging transurethral resection of the prostate in the management of stage A cancer of the prostate: a comparative evaluation. J Urol 1990;143:1142-1145

19 Gunia S, May M, Koch S, et al: MUC1 expression in incidental prostate cancer predicts staging and grading on the subsequent radical prostatectomy. Pathol Oncol Res 2010;16: 371-375.

20 Jones CU, Hunt D, McGowan DG, et al: Radiotherapy and short-term androgen deprivation for localized prostate cancer. N Engl J Med 2011;365:107-118.

21 D’Amico AV, Manola J, Loffredo M, et al: 6-month androgen suppression plus radiation therapy vs radiation therapy alone for patients with clinically localized prostate cancer: a randomized controlled trial. JAMA 2004; 292:821-827.

-22 Zelefsky MJ, Pei X, Chou JF, et al: Dose escalation for prostate cancer radiotherapy: predictors of long-term biochemical tumor control and distant metastases-free survival outcomes. Eur Urol 2011;60:1133-1139.

23 Gontero P, Kirby RS: Nerve-sparing radical retropubic prostatectomy: techniques and clinical considerations. Prostate Cancer Prostatic Dis 2005;8:133-139.
24 Lawrence EM, Gnanapragasam VJ, Priest AN, Sala E: The emerging role of diffusionweighted MRI in prostate cancer management. Nat Rev Urol 2012;9:94-101.

25 Barentsz JO, Richenberg J, Clements R, et al ESUR prostate MR guidelines 2012. Eur Radiol 2012;22:746-757.

26 Bloch BN, Genega EM, Costa DN, et al: Prediction of prostate cancer extracapsular extension with high spatial resolution dynamic contrast-enhanced 3-T MRI. Eur Radiol 2012; 22:2201-2210.

27 Sharma NL, Papadopoulos A, Lee D, et al: First 500 cases of robotic-assisted laparoscopic radical prostatectomy from a single UK centre: learning curves of two surgeons. BJU Int 2011;108:739-747.

28 Patel VR, Thaly R, Shah K: Robotic radical prostatectomy: outcomes of 500 cases. BJU Int 2007;99:1109-1112.

29 Klotz L, Zhang L, Lam A, et al: Clinical results of long-term follow-up of a large, active surveillance cohort with localized prostate cancer. J Clin Oncol 2010;28:126-131.

30 Hardie C, Parker C, Norman A, et al: Early outcomes of active surveillance for localized prostate cancer. BJU Int 2005;95:956-960.

31 Dall'Era MA, Konety BR, Cowan JE, et al: Active surveillance for the management of prostate cancer in a contemporary cohort. Cancer 2008;112:2664-2670.

32 van As NJ, Norman AR, Thomas K, et al: Predicting the probability of deferred radical treatment for localised prostate cancer managed by active surveillance. Eur Urol 2008;54: 1297-1305.
TRUSBx in Incidentally Diagnosed Prostate Cancer following TURP for BPH
Urol Int 2013;91:397-403 